\title{
MICROMECHANICAL MODELLING OF SELF-HEALING CEMENTITIOUS MATERIALS
}

\author{
R. DAVIES ${ }^{*}$ AND A. JEFFERSON ${ }^{\dagger}$ \\ * Cardiff University \\ Cardiff, UK \\ e-mail: daviesre11@,cardiff.ac.uk \\ ${ }^{\dagger}$ Cardiff University \\ Cardiff, UK \\ e-mail: jeffersonad@cardiff.ac.uk
}

Key words: Micromechanics, Self-Healing, Cementitious Materials, Cracking

\begin{abstract}
A number of numerical models have been developed for simulating self-healing behaviour, these include models based on hydration, mechanical damage at the macro-scale and coupling hydro-chemo-mechanical processes. This paper describes a new model for simulating selfhealing behaviour in cementitious materials by using a 3D two phase micro-mechanical constitutive formulation. The composite is modelled using a Mori-Tanaka homogenisation scheme and the stress concentrations, adjacent to inclusions, are included using an exterior point Eshelby solution. Anisotropic micro-cracking is simulated using arrays of circular cracks. This model incorporates self-healing by using a new solidification formulation. The focus of this paper is on how the different model parameters affect the predicted response of structural elements formed from selfhealing materials. The initiation and subsequent evolution of micro-cracks in both the original and healed materials are simulated alongside the healing processes. The performance of the 3D micromechanical self-healing model is illustrated using a series of stress-strain paths that involve damage and healing cycles. A series of paths and their stress-strain responses are shown whereby healing takes place whilst the material is subject to load. The examples show that this micromechanical self-healing model is capable of representing the characteristic mechanical response of self-healing cementitious materials with a good degree of accuracy.
\end{abstract}

\section{INTRODUCTION}

In recent years much research has been undertaken on the subject of self-healing in cementitious materials [1], [2]. This research has primarily focused on experimental work rather than simulating the material response using numerical or analytical models. Despite this trend, a number of models have been developed for simulating self-healing behaviour [3]-[10]. The majority of the mechanical healing models developed to date are phenomenological in nature and have been applied in finite element codes using the smeared crack concept. However, the present authors favour more mechanistic approach [11], [12].

One of the first self-healing models was based on thermodynamic principles which was developed by Miao et al. [13], who used an experimentally derived rate of healing function for crushed rock salt. Alfredsson \& Stigh [14], in their self-healing model for engineering materials, employed a damage variable based on an equivalent strain parameter that increased during continued damage but 
decreased when healing was simulated.

In an elastoplastic-damage-healing constitutive model for fibre reinforced polymer-matrix composites, Barbero et al. [15], used an elastic strain energy equivalence formulation to obtain a new damage-healing tensor. This concept was later extended to deal with healing in shape memory polymers [16], [17].

Abu Al-Rub et al. [18] simulated healing in asphalt, subjected to fatigue loading, by applying a healing factor to the damage variable. Their micro-damage healing model combines nonlinear viscoelastic, viscoplastic and viscodamage theories and uses a phenomenological healing evolution function. The authors subsequently generalised their micro-damage healing model by formulating it within a general thermodynamic framework [19], [20], thereby extending its range of applicability.

Many of the aforementioned constitutive models are dependent on strength properties and defined damage relationships. In cementitious materials, the development of material properties and recovery can be linked to the hydration process, particularly for early age crack healing [3], [5], [7].

Hydration processes and damage have been considered in a coupled model [10], [21] where the evolution of healing was linked to both the degree of hydration and to the value of the damage parameter at time of healing. It is noted that, in this model, the evolution of damage in the newly healed material takes precedence over primary damage, which only continues in the original material when the healed material is fully damaged.

A micro-scale model naturally captures the early stages of micro-cracking and the extent of damage in the fracture process zone around a macro-crack by considering the behaviour of the composite components. A model which can represent micro-cracks is ideally suited for including self-healing behaviour by healing these explicit cracks.

The aim of the present paper is to illustrate the predictive capabilities of the proposed new micro-mechanical self-healing model and to show how the different model parameters control the predicted response.

\section{MODEL THEORY}

\subsection{Basic constitutive model theory}

A cementitious material with aggregate particles and cement paste is represented using a two phase composite with inclusions $(\Omega)$ and a matrix (M) phase. A detailed description of the basic model can be found in Mihai \& Jefferson [11], [22] and; Davies \& Jefferson [12]. A full description of the 3D micromechanical self-healing model is presented in a forthcoming journal paper [23]. A brief summary of the model and key selfhealing components are presented in this paper.

The constitutive equation representing the relationship between the stress $(\overline{\boldsymbol{\sigma}})$ and strain $(\overline{\boldsymbol{\varepsilon}})$ is shown in equation (1). $\mathbf{D}_{M \Omega}$ is the composite elastic tensor whose properties are computed using the classical Eshelby [24] solution and the Mori-Tanaka homogenization scheme for non-dilute inclusions [25], [26]. $\boldsymbol{\varepsilon}_{\mathbf{a}}$ is the total additional strain resulting from anisotropic micro-cracking using the approach of Budiansky and O'Connell [27].

$$
\overline{\boldsymbol{\sigma}}=\mathbf{D}_{M \Omega}:\left(\overline{\boldsymbol{\varepsilon}}-\boldsymbol{\varepsilon}_{\mathrm{a}}\right)
$$

The total additional strain is obtained by integrating the added strain contributions from all directions around a hemi-sphere. This additional strain, in any one direction, is dependent on the material's Young's Modulus $(E)$, Poisson's ratio $(v)$, the density of the cracks and local equivalent local stress tensor, as given by Nemat Nasser and Hori [28]. A local stress-strain relationship for the micromechanical model is defined in equation (2), in which the added strain is taken to be the equivalent of a micro-cracked band in the material.

$$
\mathbf{s}_{L}=(1-\omega) \mathbf{D}_{L} \boldsymbol{\varepsilon}_{L}
$$

$\mathbf{s}_{L}$ is the equivalent local stress tensor and $\boldsymbol{\varepsilon}_{L}$ is the equivalent local strain tensor, both of which are expressed in a reduced vector form that considers only those components that are 
non-zero. $\mathbf{D}_{L}$ is a $3 \times 3$ matrix containing the non-zero components the local stiffness tensor. The local compliance tensor is defined as $\mathbf{C}_{L}=\mathbf{D}_{L}^{-1} . \omega$ is the micro-crack variable for each direction, taking the values between 0 for uncracked and 1 for fully micro-cracked state. The elastic local strain can be subtracted from the local strain within the micro-crack band $\left(\varepsilon_{\mathrm{L}}\right)$ to give the additional strain resulting from the crack in one direction.

\subsection{Addition of healing into the local constitutive model}

Healing is incorporated into the local constitutive relationship (i.e. for a particular direction) by restoring the stiffness of a proportion of the damaged component of material and including an offset or 'solidification' strain. The healed local stress is given in equation (3)

$$
\mathbf{s}_{L h}=(1-\omega) \mathbf{D}_{L} \boldsymbol{\varepsilon}_{L h}+h \omega_{t h} \mathbf{D}_{L h}\left(\boldsymbol{\varepsilon}_{L h}-\boldsymbol{\varepsilon}_{s}\right)
$$

The healing proportion is defined by the parameter $h$, which takes the values between 0 for no healing and 1 for fully healed. A subscript $h$ is added to the terms to show the healing equivalent terms. $\mathbf{s}_{L h}$ is the equivalent local stress tensor after healing, $\boldsymbol{\varepsilon}_{L h}$ is the local equivalent strain tensor after healing and $\omega_{t h}$ is the micro-cracking parameter at the time of healing. $\mathbf{D}_{L h}$ is the local stiffness of the healed material which can also be expressed using material parameter $B$ which is a ratio between the stiffness of the healed and original material, as show in equation (4).

$$
\mathbf{D}_{L h}=B \cdot \mathbf{D}_{L}
$$

The 'solidification strain' $\left(\boldsymbol{\varepsilon}_{\mathrm{s}}\right)$ is included to ensure that the healing material solidifies in a stress free state. The $\varepsilon_{\mathrm{s}}$ is made equal to, and fixed, at the local strain at the time of healing, which takes the form shown in equation (5).

$$
\boldsymbol{\varepsilon}_{s}=\left(\frac{1}{1-\omega_{t h}}\right) \mathbf{C}_{L}: \mathbf{s}_{L h}
$$

Furthermore, this newly healed material can also undergo micro-cracking. This is simulated by also including a healed micro-cracking variable $\omega_{h}$ into the local stress-strain relationship as shown in equation (6).

$\mathbf{s}_{L h}=(1-\omega) \mathbf{D}_{L} \boldsymbol{\varepsilon}_{L h}+\left(1-\omega_{h}\right) h \omega_{t h} B \mathbf{D}_{L}\left(\boldsymbol{\varepsilon}_{L h}-\boldsymbol{\varepsilon}_{s}\right)$

By incorporating this new healed local stress-strain relationship into the micromechanical solution the constitutive relationship is obtained as shown in equations (7) to (11). The detailed derivation contains a number of steps which are not shown here but are documented in author's forthcoming paper [23].

$$
\overline{\boldsymbol{\sigma}}=\left(\mathbf{I}^{4 s}+\mathbf{D}_{M \Omega} \mathbf{C}_{a d h \omega}\right)^{-1} \mathbf{D}_{M \Omega}:\left(\overline{\boldsymbol{\varepsilon}}-\boldsymbol{\varepsilon}_{a s \omega}\right)
$$

where,

$$
\mathbf{C}_{a d h \omega}=\frac{1}{2 \pi} \int_{2 \pi} \int_{\frac{\pi}{2}} \operatorname{Int} 1 \sin (\psi) \mathrm{d} \psi \mathrm{d} \theta
$$

with the integrated term given as,

$$
\text { Int } 1=\mathbf{N}_{\varepsilon}: \mathbf{C}_{L}: \mathbf{N} \cdot\left(\frac{\omega-\left(1-\omega_{h}\right) h \omega_{t h} B}{1-\omega+\left(1-\omega_{h}\right) h \omega_{t h} B}\right)
$$

and where

$$
\boldsymbol{\varepsilon}_{\text {as } \omega}=\left[\frac{1}{2 \pi} \int_{2 \pi} \int_{\frac{\pi}{2}} \operatorname{Int} 2 \sin (\psi) \mathrm{d} \psi \mathrm{d} \theta\right] \boldsymbol{\varepsilon}_{s}
$$

with the integrated term given as,

$$
\text { Int2 }=\mathbf{N}_{\varepsilon}:\left(\frac{\left(1-\omega_{h}\right) h \omega_{t h} B}{1-\omega+\left(1-\omega_{h}\right) h \omega_{t h} B}\right)
$$

$\mathbf{I}^{4 s}$ is the fourth order identity tensor. $\mathbf{N}_{\varepsilon}$ and $\mathbf{N}$ are the transformation tensors relating local strain and stress to the average composite strain and stress. 


\section{IMPLEMENTATION}

\subsection{Micro-crack criterion and evolution}

The original and healed micro-crack initiation and evolution criteria are based on the form adopted by Mihai \& Jefferson [11]. The onset of micro-cracking is controlled by the elastic stress field in a narrow band of material adjacent to a coarse aggregate particle. This initiation begins when the local principal stress exceeds the initial interface tensile strength. Once formed, the extent of micro-cracking is expressed in terms of the damage parameter $(\omega)$, and this relationship depends on the effective local strain parameter [11]. A detailed description of the micro-crack initiation and evolution criteria, as well as the healing functions is given in author's forthcoming paper [23].

The key parameters included in this model to control the micro-cracking are the local tensile strength $\left(f_{t}\right)$ at the aggregate/cement paste interface, the strain at first uniaxial micro-cracking $\left(\varepsilon_{t}\right)$ and an uniaxial local strain in the effectively fully micro-cracked state $\left(\varepsilon_{0}\right)$.

\subsection{Numerical algorithms}

The self-healing micromechanical model has been implemented in a Mathcad [29] sheet using a constitutive driver algorithm. The model follows a specified path, which is defined by stress and/or strain increments (i.e. $\Delta \boldsymbol{\sigma}, \Delta \boldsymbol{\varepsilon}$ respectively) [30]. The simulation considers the period of time before healing occurs ( $t_{0}$ to $\left.t_{\text {heal }}\right)$, the moment of healing ( $\left.t_{\text {heal }}\right)$ as well as the post-healing phase ( $t_{\text {heal }}$ to $\left.t_{n s}\right)$. The full 3D model, with 29 sample directions for the spherical integrals is used for all simulations.

\section{CHARACTERISTIC MODEL PREDICTIONS}

\subsection{Material properties}

This parametric study considers the stress strain response of the material when varying the local tensile strength $\left(f_{t h}\right)$, the local strains $\left(\varepsilon_{0 h}\right)$ in the effectively fully micro-cracked state, healing efficiency $(h)$ and strength of the healed material $(B)$. The material properties used during this parametric study are shown in Table 1. The properties are varied one at time using ratio $p$ which takes the values $0.25,0.5$, 1 and 2 . The same micro-cracking function is used for both original and healed material.

Table 1: Material properties used in model.

\begin{tabular}{|c|c|c|c|c|}
\hline$f_{M}$ & $\begin{array}{c}E_{M} \\
(\mathrm{kN} / \\
\left.\mathrm{mm}^{2}\right)\end{array}$ & $v_{M}$ & $\begin{array}{c}f_{t} \\
(\mathrm{kN} / \\
\left.\mathrm{mm}^{2}\right)\end{array}$ & $\begin{array}{c}f_{\text {th }} \\
(\mathrm{kN} / \\
\left.\mathrm{mm}^{2}\right)\end{array}$ \\
\hline 0.3 & 20 & 0.15 & 1 & $p \cdot f_{t}$ \\
\hline$\overline{f_{\Omega}}$ & $\begin{array}{c}E_{\Omega} \\
(\mathrm{kN} / \\
\left.\mathrm{mm}^{2}\right)\end{array}$ & $\overline{v_{\Omega}}$ & $\begin{array}{c}\varepsilon_{t} \\
10^{-5}\end{array}$ & $\begin{array}{c}\varepsilon_{t h} \\
10^{-5}\end{array}$ \\
\hline 0.7 & 55 & 0.21 & 5 & \\
\hline$\varepsilon_{0}$ & $\varepsilon_{0 h}$ & $h$ & $B$ & \\
\hline 0.067 & $p \cdot E_{0}$ & $p \cdot 0.5$ & $p \cdot 0.5$ & \\
\hline
\end{tabular}

$f_{M}$ and $f_{\Omega}$ are the volume fractions of the matrix and inclusion material respectively.

\subsection{Model response}

A cyclic uniaxial tensile strain path, shown in Figure 1, is considered for the reference material parameters and the resulting strainstress response is shown in Figure 2. The sample is loaded linearly with respect to time between points $a$ and $b$, and then unloaded between points $\mathrm{b}$ and $\mathrm{c}$. The healing is assumed to take place at point $\mathrm{c}$ when the $\mathrm{x}-\mathrm{x}$ strain component $\left(\bar{\varepsilon}_{x x}\right)$ is zero. $\bar{\varepsilon}_{x x}$ is increased linearly during the reloading phase, during which the stress response is shown to have two distinct gradients up to the peak post-healing stress. The steep gradient, at the start of this loading phase, reflects the stiffness of the healed material, whilst the flatter gradient of the following section results from a combination of micro-cracking in the newly healed material and the original material taking stress without further micro-cracking. In the final section, at point $d$, the level of micro-cracking is increasing in both the newly healed and original material. 


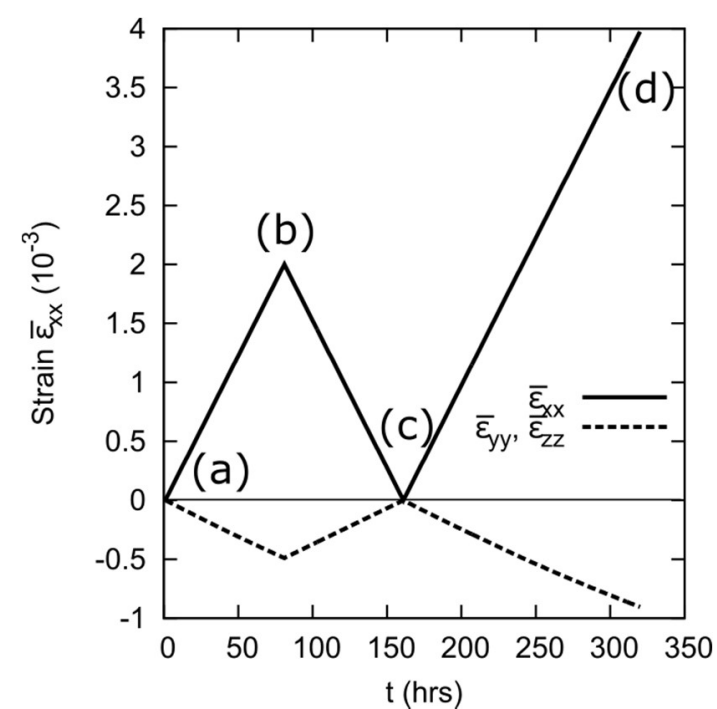

Figure 1: Uniaxial tensile strain path.

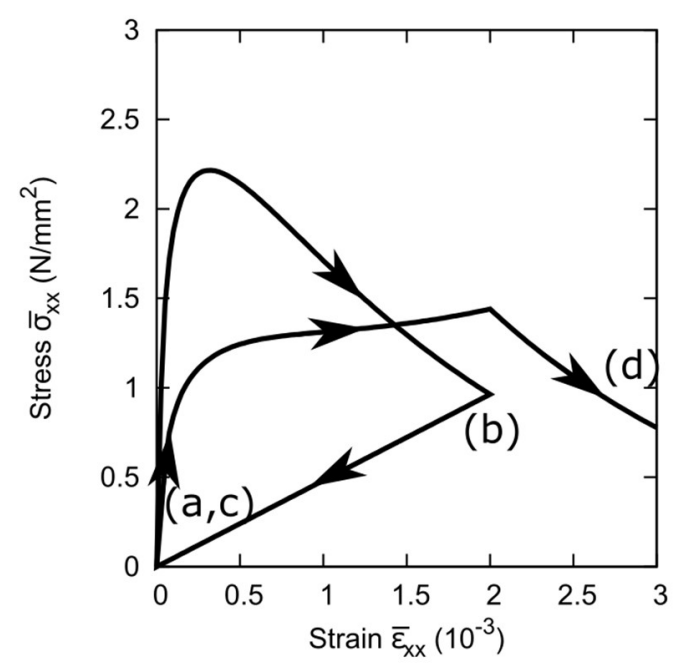

Figure 2: Strain stress response.

\subsection{Varying healed material local strength}

Figure 3 shows the influence of changing the ratio between $f_{t}$ and $f_{t h}$ on the strain-stress response. This ratio controls the stress of the first portion of the post-healing linear reloading phase linked to the initial microcracking of the new material. This ratio also controls the peak reloading stress of the healing recovery. The immediate post-healing stiffness increases with $f_{t h}$. It is noted that since $f_{t}$ remains constant for all analysis, the pre-healing response (i.e. a to c) is the same for all cases.

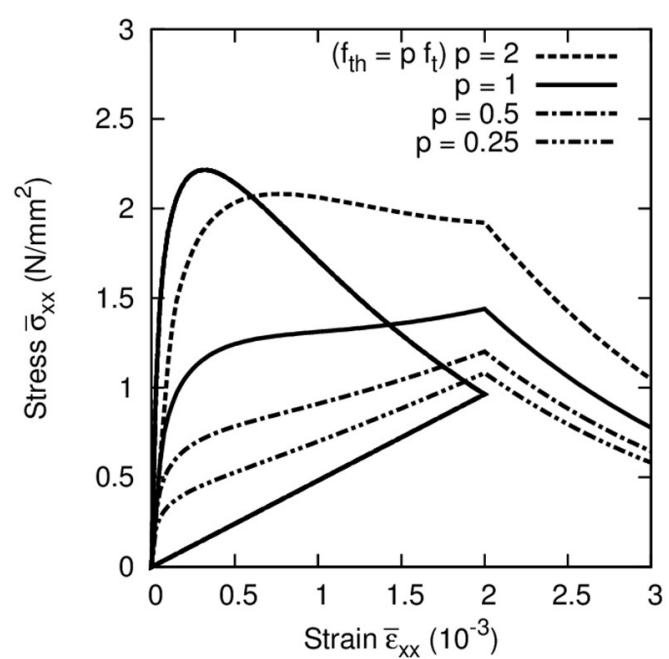

Figure 3: Varying healed local strength $\left(f_{t h}\right)$.

\subsection{Varying healed effective uniaxial strain}

Figure 4 shows how the fully microcracked local strain of the healed material influences the material response. The larger the $\varepsilon_{0} h$ value the less the healed material micro-cracks. It can be seen that for a smaller $\varepsilon_{0 h}$ the new healed material is almost fully micro-cracked by the time the composite reaches the original unloading strain, as shown by the plot of $p=0.25$, resulting in negligible changes to the healing recovery.

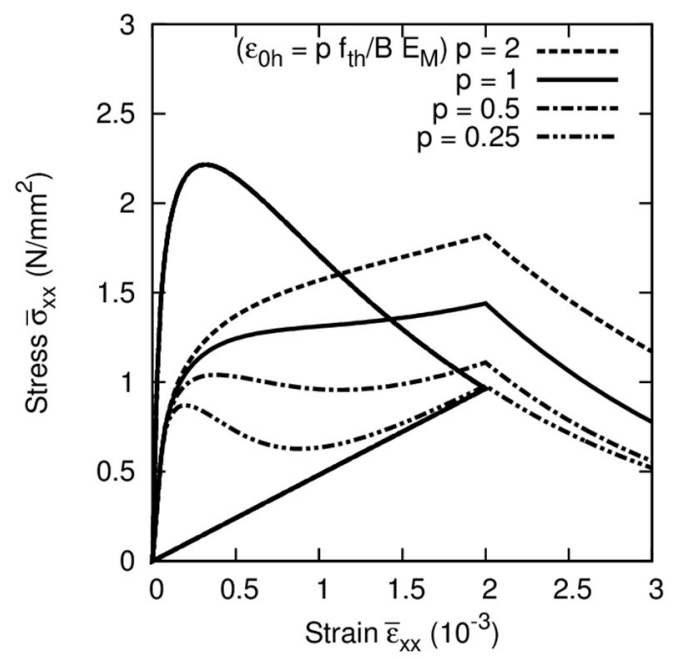

Figure 4: Varying effective uniaxial strain $\left(\varepsilon_{0 h}\right)$.

\subsection{Varying healed efficiency}

Figure 5 shows how the healing efficiency (h) influences the response of the material. The initial change in gradient during reloading and the healing efficiency are both affected with 
the change in $h$. it is noted that the model is particularly sensitive to the change in $h$.

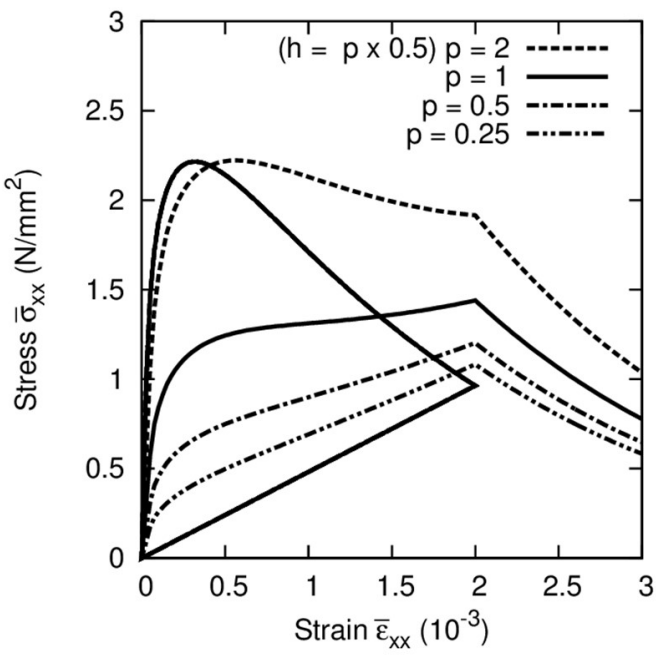

Figure 5: Varying healed efficiency $(h)$.

\subsection{Varying healed strength ratio}

Changing the relative stiffness of the healed material $(B)$, has minimal impact on the response of the model, as shown in Figure 6.

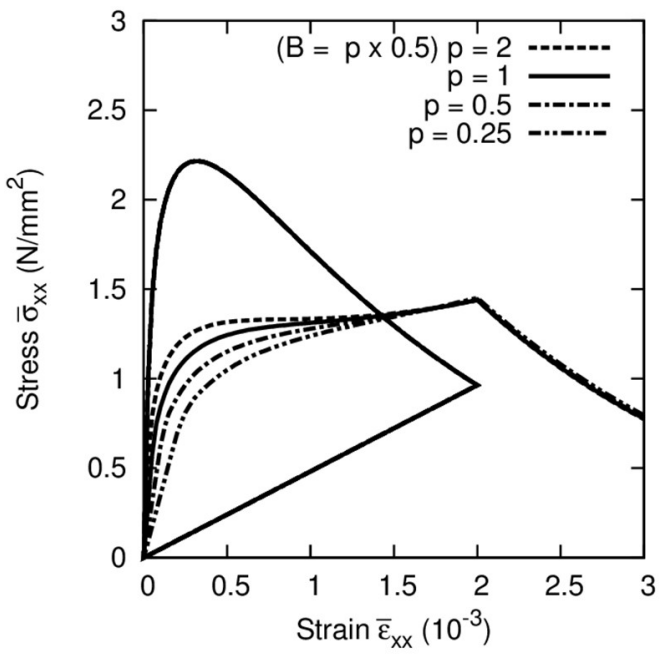

Figure 6: Varying healing strength ratio $(B)$.

\section{HEALING UNDER LOADED CONDITIONS}

The parametric study, in the previous section, showed healing taking place when there is zero applied load and strain. This means that the $\boldsymbol{\varepsilon}_{\mathrm{s}}$, from equation (5), was zero and $\boldsymbol{\varepsilon}_{a s \omega}$ from equation (10) also remained zero. Real structures are likely to undergo healing whilst being subjected to self-weight and some serviceability loading. Therefore, the predicted healing response of the model in which healing takes place under loaded conditions is examined in this section.

A series of paths have been chosen whereby the healing takes place at different strains during the unloading phase. Figure 7 shows five paths with uniaxial strain applied in the $\mathrm{x}$ $\mathrm{x}$ direction. $100 \%$ unloading takes place in Path 1 (similar to the parametric study) and the degree of unloading is reduced in Paths 2 to 4, eventually showing no unloading in Path 5. The composite is strained to a fixed value in Path 5 where healing takes place instantaneously before then continuing the uniaxial strain at the same initial rate. The strain-stress response of the model for these different paths are shown in Figure 8.

An interesting feature of the responses can be seen when plotting all of the strain-stress paths together. The highest peak stress -during a reloading phase- occurs in Path 3 (reloaded $\left.0.5 \bar{\sigma}_{x x \_ \text {unload }}\right)$, with the true maximum occurring for a path intermediate between Path 3 and Path 4. During the reloading phase, micro-cracking progresses in both the undamaged material and in the healed material, and it seems that the balance between these two damage processes results in a reload maximum at approximately $0.6 \bar{\sigma}_{x x \_ \text {unload }}$.

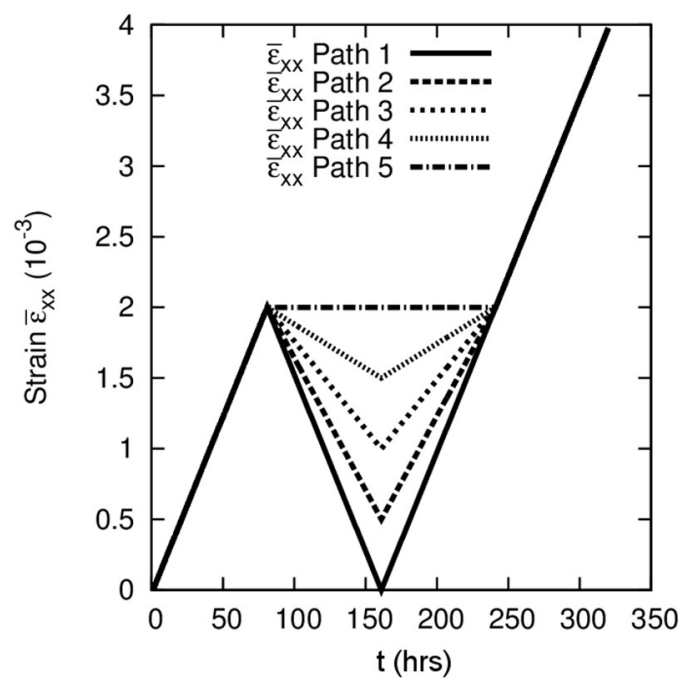

Figure 7: Uniaxial tensile strain path with healing under load. 


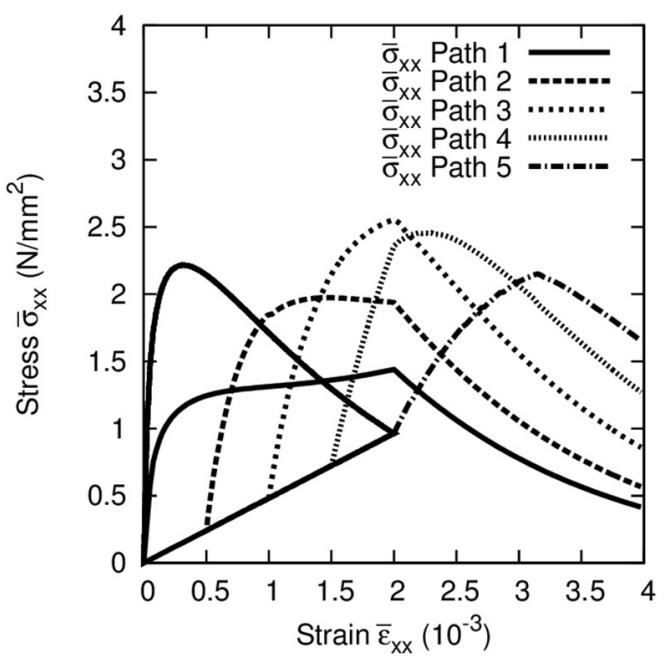

Figure 8: Strain stress response - healing under load.

\section{CONCLUSIONS}

A two phase composite micro-mechanical constitutive model, that includes anisotropic micro-cracking, provides an excellent basis for the development of a model for cementitious materials that includes self-healing behaviour. The relative simplicity of this micromechanical healing model combined with the fact that it requires a small number of physically meaningful parameters shows that it is suitable for simulating a wide range of twophase cementitious materials.

The new micro-mechanical model can simulate the characteristic damage-healing behaviour typically observed for cementitious materials with partial or complete recovery of strength and stiffness. The continued damage (or micro-cracking) of a healed region is achieved. The healing material forms in a stress free state such that the stress state does not change at the instant of healing. The ability to simulate healing under both loaded and unloaded conditions allows the model to simulate realistic loading conditions.

\section{AKNOWLEDGEMENTS}

Thanks must go to EPSRC for their funding of the Materials for Life (M4L) project (EP/K026631/1).

\section{REFERENCES}

[1] M. R. de Rooij, K. Van Tittelboom, N. De Belie, and E. Schlangen, Self-Healing
Phenomena in Cement-Based Materials: State-of-the-Art Report of RILEM Technical Committee 221-SHC: SelfHealing Phenomena in Cement-Based Materials. Bagneux, France: Springer, 2013.

[2] K. Van Tittelboom and N. De Belie, 'Self-Healing in Cementitious Materials-A Review', Materials, vol. 6, no. 6, pp. 2182-2217, May 2013.

[3] E. Schlangen, N. Ter Heide, and K. Van Breugel, 'Crack healing of early age cracks in concrete', in ECF16, Alexandroupolis, Greece, 2006.

[4] E. C. Schimmel and J. J. C. Remmers, 'Development of a constitutive model for self-healing materials', Delft Aerospace Computational Science, DACS-06-003, 2006.

[5] G. Ye and K. van Breugel, 'Potential use of HYMOSTRUC cement hydration model for self-healing of microcracks in cementitious materials', in Proceedings of the First International Conference on Self Healing Materials, Noordwijk aan Zee, The Netherlands, 2007.

[6] J. J. Remmers and R. de Borst, 'Numerical Modelling of Self Healing Mechanisms', in Self Healing Materials, Springer, 2008, pp. 365-380.

[7] H. Huang and G. Ye, 'Simulation of selfhealing by further hydration in cementitious materials', Cem. Concr. Compos., vol. 34, no. 4, pp. 460-467, Apr. 2012.

[8] M. K. Darabi, R. K. Abu Al-Rub, and D. N. Little, 'A continuum damage mechanics framework for modeling micro-damage healing', Int. J. Solids Struct., vol. 49, no. 3-4, pp. 492-513, Feb. 2012.

[9] J. Mergheim and P. Steinmann, 'Phenomenological modelling of selfhealing polymers based on integrated healing agents', Comput. Mech., vol. 52, no. 3, pp. 681-692, Sep. 2013.

[10] B. Hilloulin, F. Grondin, M. Matallah, and A. Loukili, 'Modelling of autogenous healing in ultra high performance 
concrete', Cem. Concr. Res., vol. 61-62, pp. 64-70, Jul. 2014.

[11] I. C. Mihai and A. D. Jefferson, 'A material model for cementitious composite materials with an exterior point Eshelby microcrack initiation criterion', Int. J. Solids Struct., vol. 48, no. 24, pp. 3312-3325, Dec. 2011.

[12] R. Davies and A. Jefferson, 'The simulation of inelastic matrix strains in cementitious materials using micromechanical solutions', Eng. Fract. Mech., 2014.

[13] S. Miao, M. Wang, and H. Schreyer, 'Constitutive Models for Healing of Materials with Application to Compaction of Crushed Rock Salt', J. Eng. Mech., vol. 121, no. 10, pp. $1122-$ 1129, 1995.

[14] K. S. Alfredsson and U. Stigh, 'Continuum damage mechanics revised: A principle for mechanical and thermal equivalence', Int. J. Solids Struct., vol. 41, no. 15, pp. 4025-4045, Jul. 2004.

[15] E. J. Barbero, L. Elgileerirlg, F. Greco, P. Lon'etti, and E. J, 'Continuum Damagehealing.Mechanics with Application to.Self-healing Composites', Int. J. Damage Mech., vol. 14, pp. 51-81, Jan. 2005.

[16] G. Z. Voyiadjis, A. Shojaei, and G. Li, 'A thermodynamic consistent damage and healing model for self healing materials', Int. J. Plast., vol. 27, no. 7, pp. 1025-1044, Jul. 2011.

[17] G. Z. Voyiadjis, A. Shojaei, G. Li, and P. Kattan, 'Continuum damage-healing mechanics with introduction to new healing variables', Int. J. Damage Mech., vol. 21, no. 3, pp. 391-414, 2012.

[18] R. K. Abu Al-Rub, M. K. Darabi, D. N. Little, and E. A. Masad, 'A microdamage healing model that improves prediction of fatigue life in asphalt mixes', Int. J. Eng. Sci., vol. 48, no. 11, pp. 966-990, Nov. 2010.

[19] R. K. Abu Al-Rub and M. K. Darabi, 'A thermodynamic framework for constitutive modeling of time- and rate- dependent materials. Part I: Theory', Int. J. Plast., vol. 34, pp. 61-92, Jul. 2012.

[20] M. K. Darabi, R. K. Abu Al-Rub, E. A. Masad, and D. N. Little, 'Constitutive modeling of fatigue damage response of asphalt concrete materials with consideration of micro-damage healing', Int. J. Solids Struct., vol. 50, no. 19, pp. 2901-2913, Sep. 2013.

[21] S. Granger, G. Pijaudier-Cabot, and A. Loukili, 'Mechanical behavior of selfhealed Ultra High Performance Concrete: from experimental evidence to modeling', Proc Fram., vol. 6, 2007.

[22] I. C. Mihai and A. D. Jefferson, 'Smoothed contact in a micromechanical model for cement bound materials', Comput. Struct., vol. 118, pp. 115-125, Mar. 2013.

[23] R. E. Davies and A. D. Jefferson, 'Micromechanical modelling of selfhealing cementitious materials', Under review, submitted Dec. 2015.

[24] J. D. Eshelby, 'The determination of the elastic field of an ellipsoidal inclusion, and related problems', Proc. R. Soc. Lond. Ser. Math. Phys. Sci., vol. 241, no. 1226, pp. 376-396, 1957.

[25] T. Mori and K. Tanaka, 'Average stress in matrix and average elastic energy of materials with misfitting inclusions', Acta Metall., vol. 21, no. 5, pp. 571-574, 1973.

[26] Y. Benveniste, 'A new approach to the application of Mori-Tanaka's theory in composite materials', Mech. Mater., vol. 6, no. 2, pp. 147-157, Jun. 1987.

[27] B. Budiansky and R. O'Connell, 'Elasticmoduli of a cracked solid', Int. J. Solids Struct., vol. 12, no. 2, pp. 81-97, 1976.

[28] S. Nemat-Nasser and M. Hori, Micromechanics: overall properties of heterogeneous materials. Amsterdam; New York: Elsevier, 1999.

[29] Mathcad, Mathcad help manual. PTC, 2010.

[30] R. E. Davies, 'Micromechanical modelling of self-healing cementitious materials', Thesis, Cardiff University, 2014. 